\title{
Identification of red high proper-motion objects in Tycho-2 and 2MASS catalogues using Virtual Observatory tools
}

\author{
F. M. Jiménez-Esteban ${ }^{1,2,3}$, J. A. Caballero ${ }^{1}$, R. Dorda ${ }^{4,5}$, P. A. Miles-Páez ${ }^{4,6}$, and E. Solano ${ }^{1,2}$ \\ ${ }^{1}$ Centro de Astrobiología (INTA-CSIC), Departamento de Astrofísica, PO Box 78, 28691 Villanueva de la Cañada, Madrid, Spain \\ e-mail: fran.jimenez-esteban@cab.inta-csic.es \\ 2 Spanish Virtual Observatory, Spain \\ 3 Saint Louis University, Madrid Campus, Division of Science and Engineering, Avenida del Valle 34, 28003 Madrid, Spain \\ ${ }^{4}$ Departamento de Astrofísica y Ciencias de la Atmósfera, Facultad de Ciencias Físicas, Avenida Complutense s/n, 28040 Madrid, \\ Spain \\ 5 Departamento de Física, Ingeniería de Sistemas y Teoría de la Señal, Escuela Politécnica Superior, University of Alicante, Apdo. 99, \\ 03080 Alicante, Spain \\ ${ }^{6}$ Instituto de Astrofísica de Canarias, 38200 La Laguna, Tenerife, Spain
}

Received 1 November 2011 / Accepted 16 January 2012

\section{ABSTRACT}

\begin{abstract}
Aims. With available Virtual Observatory tools, we looked for new M dwarfs in the solar neighbourhood and M giants with high tangential velocities.

Methods. From an all-sky cross-match between the optical Tycho-2 and the near-infrared 2MASS catalogues, we selected objects

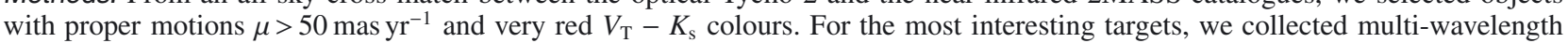
photometry, constructed spectral energy distributions, estimated effective temperatures and surface gravities from fits to atmospheric models, performed time-series analysis of ASAS $V$-band light curves, and assigned spectral types from low-resolution spectroscopy obtained with CAFOS at the $2.2 \mathrm{~m}$ Calar Alto telescope.

Results. We got a sample of 59 bright red high proper-motion objects, including fifty red giants, four red dwarfs, and five objects reported in this work for the first time. The five new stars have magnitudes $V_{\mathrm{T}} \approx 10.8-11.3 \mathrm{mag}$, reduced proper motions midway between known dwarfs and giants, near-infrared colours typical of giants, and effective temperatures $T_{\text {eff }} \approx 2900-3400 \mathrm{~K}$. From our time-series analysis, we discovered a long secondary period in Ruber 4 and an extremely long primary period in Ruber 6 . With the CAFOS spectra, we confirmed the red giant nature of Ruber 7 and 8, the last of which seems to be one of the brightest metal-poor $M$ giants ever identified.
\end{abstract}

Key words. stars: oscillations - stars: late-type - stars: chemically peculiar - virtual observatory tools - stars: peculiar - astronomical databases: miscellaneous

\section{Introduction}

For the Virtual Observatory (VO), there are seductive mottoes (e.g. the Universe at your fingertips; $\mathrm{NVO}^{1}$ ) and bombastic definitions (e.g. an international astronomical community-based initiative [that] aims to allow global electronic access to the available astronomical data archives of space and ground-based observatories and other sky survey databases [and] to enable data analysis techniques through a coordinating entity that will provide common standards, wide-network bandwidth, and stateof-the-art analysis tools; $\mathrm{EURO}-\mathrm{VO}^{2}$ ). One of the most tangible results of the VO endeavour is a suite of analysis tools (the socalled "VO tools"), which are used more and more frequently by the astronomical community, as demonstrated by the growing number of VO papers ${ }^{3}$ (see, for instance, Caballero 2009, for a good example of this).

While there is a current trend to discovering the "faintest, coolest, smallest" objects in our Galaxy (e.g. Delorme et al. 2008; Kirkpatrick et al. 2011), there is still a lot to do with relatively bright unknown objects $(V \gtrsim 11 \mathrm{mag}-$ i.e. visible through small amateur telescopes). With Caballero \& Solano (2008) and

\footnotetext{
1 http://www.us-vo.org

2 http://www. euro-vo.org

${ }^{3}$ http://www . euro-vo.org/pub/fc/papers.html
}

Jiménez-Esteban et al. (2011), we started a project devoted to identifying bright blue objects in the Tycho- 2 catalogue interesting for other follow-up studies. In particular, our surveys have provided some of the brightest hot subdwarfs ever found, which enormously facilitates forthcoming astroseismologic, spectroscopic, and multiplicity analyses (Oreiro et al. 2011; Vennes et al. 2011).

Here we go on examining high proper-motion objects in Tycho-2 and 2MASS catalogues using VO tools, this time to the other side of the colour-magnitude diagram, looking for bright red objects. In the absence of discs or dust envelopes, red colours are synonymous with low effective temperatures $\left(T_{\text {eff }} \sim 3000 \mathrm{~K}\right.$ for the coolest stars in Tycho-2). A priori, there are not many alternatives for classifying such bright late-type objects with high proper-motion: they can be either nearby $\mathrm{M}$ dwarfs or red giants with high tangential velocities.

Finding a single uncatalogued Tycho-2 dwarf later than M3V would justify the whole current survey. With $V \sim 11 \mathrm{mag}$, a star like that would have $J \lesssim 7$ mag and inevitably be a golden target for the next high-resolution, near-infrared (NIR), radialvelocity surveys seeking Earth-like planets (Reiners \& Basri 2010; Mahadevan et al. 2010; Quirrenbach et al. 2010). Such a finding was also one of the aims of Lépine \& Gaidos (2011), who present an all-sky catalogue of M dwarf stars with apparent 


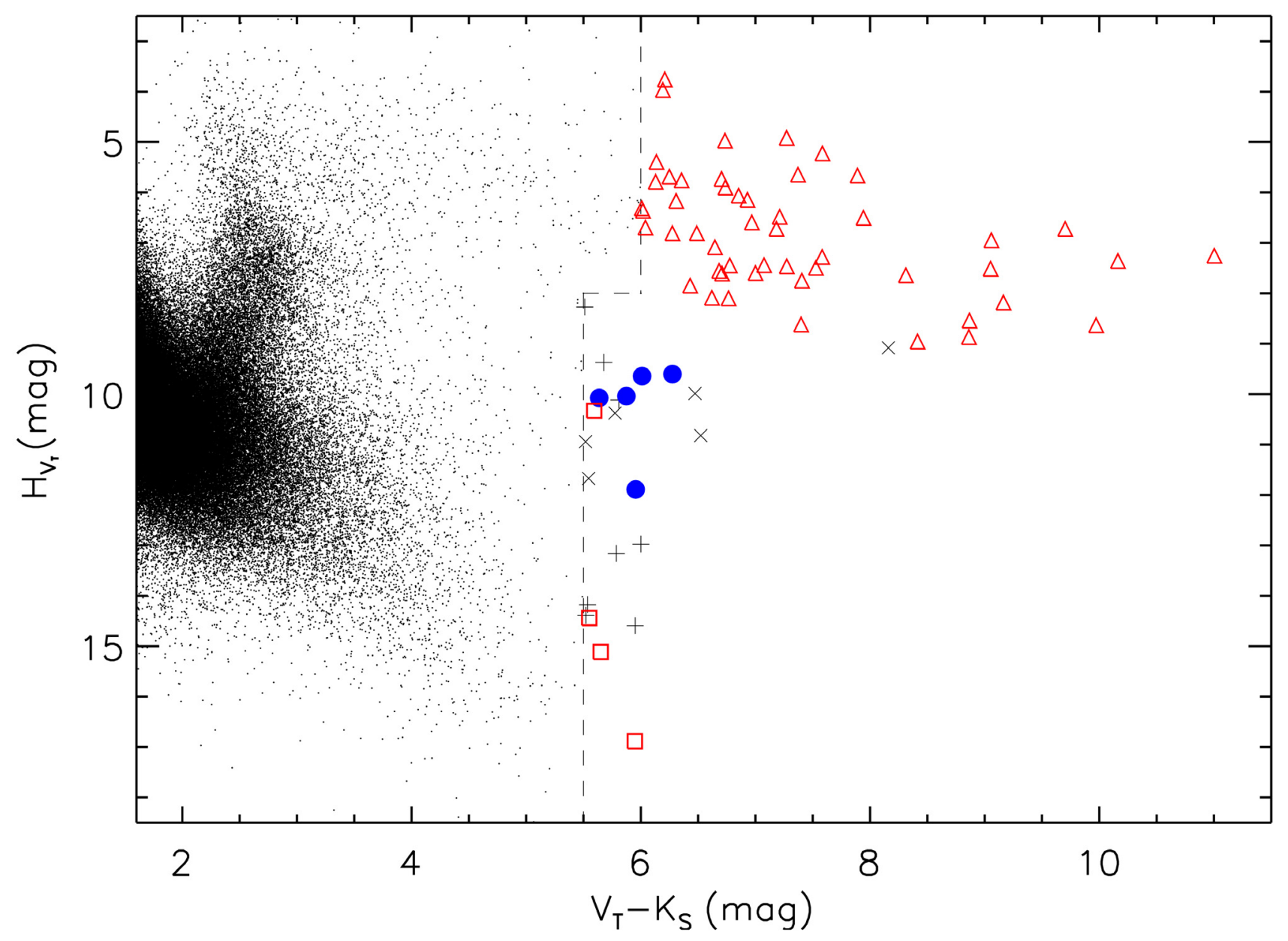

Fig. 1. Tycho-2/2MASS reduced proper motion diagram $\left(H_{V_{\mathrm{T}}}\right.$ versus $V_{\mathrm{T}}-K_{\mathrm{S}}$, where $\left.H_{V_{\mathrm{T}}}=V_{\mathrm{T}}+5 \log \mu+5\right)$. Objects selected for follow-up are redwards of the dashed line. Previously unreported red high proper-motion objects are depicted with [blue] filled circles, already-known objects with [red] open symbols (giants with triangles, dwarfs with squares), binary systems unresolved by 2MASS with [black] crosses, and objects with wrong proper motion with [black] sails. The remaining objects bluewards of the dashed line are marked with small dots. The dwarf located halfway between giants (to the top) and the remaining dwarfs (to the bottom) is BD-21 1074 BC. Compare this diagram with the one in Fig. 2 of Jiménez-Esteban et al. (2011).

infrared magnitudes $J<10.0 \mathrm{mag}$. In a sense, the Lépine \& Gaidos (2011) catalogue and ours are complementary.

The other alternative is a late-type giant (spectral class M, $\mathrm{C}$, or S). Since giants are intrinsically luminous, they are commonly located at long heliocentric distances. However, if they have appreciable proper motions, of over a few tens of milliarcseconds per year, their tangential velocities must be very high. As a result, they may belong to the Galactic thick disc or even the halo (Carney \& Latham 1986; Rocha-Pinto et al. 2004; Famaey et al. 2005). Our work is expected to catalogue all the brightest, reddest giants over the whole sky, which are excellent targets for investigating the dynamics and metallicity structure of the Milky Way (McWilliam \& Rich 1994; Lambert et al. 1986; Beers et al. 2002) and their interiors through pulsation analyses (Wood \& Cahn 1977; Bergeat et al. 2002; Kiss \& Bedding 2003). Besides this, we also expect to discover rare examples of bright red giants.

\section{Analysis and results}

\subsection{Target selection}

We used the sample of 155384 sources with proper motions $\mu>50$ mas a $^{-1}$ obtained from the cross-match of the whole
Tycho-2 (Høg et al. 2000) and 2MASS (Skrutskie et al. 2006) catalogues constructed by Jiménez-Esteban et al. (2011). We selected red high proper-motion star candidates based on their position on the reduced proper motion-colour diagram shown in Fig. 1. Quantitatively, the applied selection criterion was $V_{\mathrm{T}}-K_{\mathrm{S}}>6.0 \mathrm{mag}$ if $H_{V_{\mathrm{T}}} \leq 8 \mathrm{mag}$ and $V_{\mathrm{T}}-K_{\mathrm{s}}>5.5 \mathrm{mag}$ if $H_{V_{\mathrm{T}}}>8 \mathrm{mag}$. The abnormally large errors in $K_{\mathrm{S}}$ because of saturation of the brightest objects barely affect the data point location in the diagram. The application of this simple selection criterion resulted in 73 objects with redder colours than these values, which is an appropriate number of sources to be studied individually on a reasonable time scale.

Next, we carried out a visual inspection of the 73 selected candidates in a similar way to the one described in Jiménez-Esteban et al. (2011). Six of them were found to have erroneous proper motions in the Tycho- 2 catalogue. As an example, the catalogue gives a proper motion $\left(\mu_{\alpha} \cos \delta, \mu_{\delta}\right)=$ $(-14,-84)$ mas a $^{-1}$ for the star TYC $6238-480-1$, while we measured $(+0.9 \pm 1.4,-12.4 \pm 0.6)$ mas a $^{-1}$ using six astrometric epochs covering 43.7 years and the method exposed by Caballero (2010). In this case, the Tycho-2 measurement was likely affected by a visual (unbound) companion at 8 arcsec to the North, $\sim 2.8$ mag fainter in the $R$ band. The five other discarded stars have even lower actual proper motions. In addition, 
we discarded another eight close binary stars, resolved by Tycho- 2 but unresolved by $2 \mathrm{MASS}$, because of their incorrect resulting colours.

\subsection{Preliminary target classification}

Of the 59 remaining unresolved objects, 54 have some kind of published spectral type information. We list their basic properties in Tables 1 and 2, where we provide their Tycho-2 identification, coordinates, Tycho- $2 V_{\mathrm{T}}$ and $2 \mathrm{MASS} J H K_{\mathrm{s}}$ magnitudes, total proper motion $\mu$, parallax (from van Leeuwen 2007 in all but two dwarfs), spectral type, most common name, and at least one of the most representative references.

Most of the known reddest Tycho-2/2MASS stars with $\mu>50$ mas a $^{-1}$ are giants. Except for a few cases, the fifty stars in Table 1 are well known class-III giants (such as Mira Cet AB, $\mathrm{L}_{2}$ Pup, $g$ Cen, and R Lyr, which are the only stars in the table at $d<100 \mathrm{pc}$ ), semi-regular pulsating, irregular, or Mira variables. They are located at long parallactic distances, have strong flux densities in the InfraRed Astronomical Satellite (IRAS) catalogue of point sources, saturate in 2MASS $J H K_{\mathrm{s}}$ bands, and/or already have spectral type and class determinations (mostly found in the spectral type compilation by Kwok et al. 1997) consistent with its location in the reduced proper motion diagram far from the main sequence. The only exceptions to the collection of stars with evidence of "giantism" are the poorly investigated stars BD+31 1540 and HD 150184. The former was already presented as a star with a remarkable spectrum by Espin (1892) in the 19th century, and it has the same properties as the rest of the stars in the table. The latter does not have a SIMBAD entry at all, but was tabulated as an M3-4 giant star by Houk \& Cowley (1975).

The four other known stars, shown in Table 2, are M dwarfs in the solar neighbourhood. While two of them have accurate parallactic distances measured by HIPPARcos (QY Aur AB: $d=$ $6.29 \pm 0.12$ pc; YZ CMi: $d=5.95 \pm 0.07$ pc; van Leeuwen 2007), the other two only have photometric distances placing them at 810 pc (Reid et al. 1995; Beuzit et al. 2004). Three of the four stars have proper motions larger than any giant in Table 1, and one of them up to 1000 mas a $^{-1}$. The four stars have spectral type determinations at $\mathrm{M} 3.5-4.5 \mathrm{~V}$, consistent with the observed optical and NIR colours (in contrast to the giants, none of the dwarfs saturate in the 2MASS $J H K_{\mathrm{s}}$ bands). Interestingly, the four dwarfs are (candidate) members in multiple systems and/or young moving groups:

- BD-21 1074 BC is part of a hierarchical triple system, WDS 05069-2135, which was discovered by Donner (1935). The primary (A, GJ 3331) is a nearby flare M1.5V star at 8.22 arcsec to a tighter system (BC, GJ 3332) of two slightly cooler stars separated by only 0.80 arcsec. Neither Tycho2 nor 2MASS were able to resolve the tight binary, which is 1.15 mag redder than the primary in $V_{\mathrm{T}}-K_{\mathrm{s}}$. Based on a significant amount of $\mathrm{Li} \mathrm{I}$ in absorption in the spectra of both A and BC components, X-ray emission, and kinematics, da Silva et al. (2009) classified the system as a member of the very young $\beta$ Pictoris association $(\tau \sim 12 \mathrm{Ma})$.

- QY Aur AB is a "classic" double in the solar neighbourhood. It is a spectroscopic binary with an approximate period of $10.43 \mathrm{~d}$ and a relatively high orbital eccentricity of 0.34 with no previous report of membership in a young moving group.

- YZ CMi, although it has a Washington Double Star catalogue entry, seems to be single (Lafrenière et al. 2007). It is an emission-line dwarf with megaflares, which has been repeatedly investigated (e.g., Kunkel 1969; Kahler et al. 1982; Kundu et al. 1988; Benz \& Alef 1991). Montes et al. (2001) classify the star as a member of the Local Association $(\tau \sim 10-150 \mathrm{Ma})$.

- The magnetically active binary star DG CVn AB (WDS 13318+2917), one of the only three known M dwarf ultra-fast rotators within $10 \mathrm{pc}$ (Delfosse et al. 1998), was first resolved by Beuzit et al. (2004) into a pair separated by only 0.17 arcsec. Montes et al. (2001) classified the binary as a member of the young disc $(\tau \sim 600 \mathrm{Ma})$.

The remaining five unknown stars, listed in Table 3 and plotted in Fig. 1 with filled (blue) circles, have no Hipparcos entry and have never been reported in the literature. Actually, one of them has a Bonner Durchmusterung entry (Schönfeld 1886), but it has gone without being noticed for over 120 years. For naming the stars, we followed the "Ruber" (red in Latin) nomenclature introduced by Caballero \& Solano (2008) and followed by Caballero (2009). Our new Ruber objects go from the fourth to the eighth in this series.

The five new red high proper-motion objects fall between the giant and (ordinary) dwarf branches in the reduced proper motion-colour diagram. Are the Ruber objects unresolved binary dwarfs in the $\beta$ Pictoris moving group, such as BD-21 1074 BC, or peculiar underluminous giants? To shed some light on their nature, we first analysed their NIR colours.

Figure 2 shows the location of our sources in an $H-K_{\mathrm{s}}$ vs. $J-$ $H$ colour-colour diagram, which is an excellent diagnostic tool for distinguishing between red giants and dwarfs (Bessell \& Brett 1988). All fifty M giants in Table 1 are clearly segregated from the four $\mathrm{M}$ dwarfs in Table 2, which match their expected intrinsic colours. The five new Ruber objects are located in the giant region, indicating that they are probably $\mathrm{M}$ giants with very high tangential velocities.

\subsection{Virtual observatory analysis}

\subsubsection{Spectral energy distributions}

Another way to obtain information of the Ruber objects is by analysing their spectral energy distributions (SEDs). We searched for additional photometric data of the five Ruber objects using the "all-VO Discovery tool" of Aladin sky atlas (Bonnarel et al. 2000). This utility allows the user to query a large number of photometric catalogues in a convenient way. Besides Tycho-2 and 2MASS, we collected observational data from the following astrophotometric catalogues: UCAC3 (Zacharias et al. 2010), DENIS (Epchtein et al. 1997), WISE (Wright et al. 2010), GLIMPSE (Churchwell et al. 2009), MSX6C (Egan et al. 2003), AKARI/IRC (Ishihara et al. 2010), and IRAS (Beichman et al. 1988). Table 3 compiles all this information.

We took advantage of another VO tool, $\operatorname{VOSA}^{4}$ (VO SED Analyzer; Bayo et al. 2008), to fit the observed SEDs to theoretical models available at the VO. VOSA allows the user to query, in an automatic and transparent way, different collections of theoretical models, calculate their synthetic photometry, and perform a statistical test to determine which model reproduces the observed data best.

To fit the SEDs of our Ruber objects, we used the NextGen collection of stellar atmosphere models (Hauschildt et al. 1999) and solar metallicity. The effective temperatures $\left(T_{\text {eff }}\right)$ and surface gravities $(\log g)$ obtained with VOSA ranged between 2900

${ }^{4}$ http://svo.cab.inta-csic.es/theory/vosa 
A\&A 539, A86 (2012)

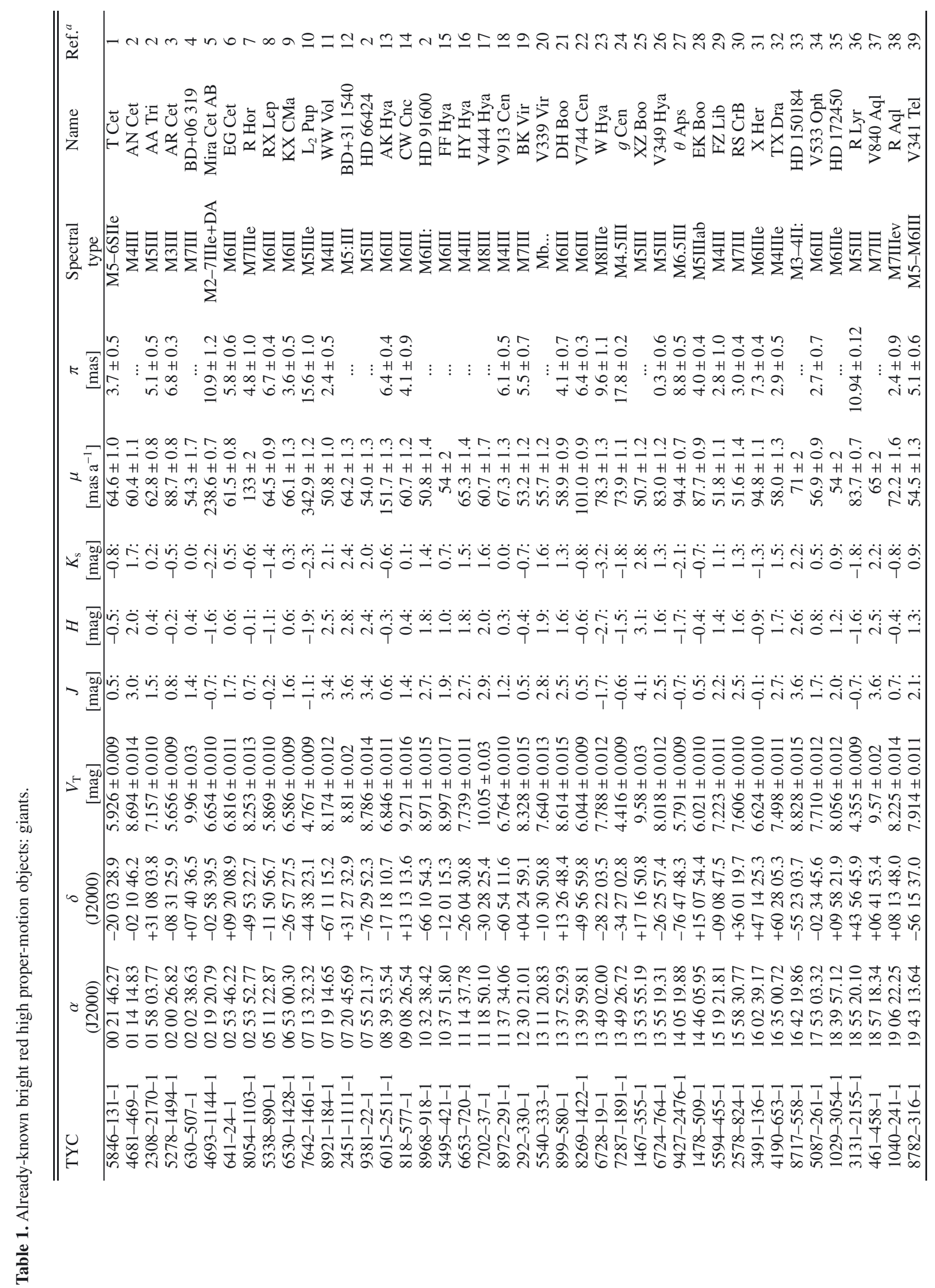


F. M. Jiménez-Esteban et al.: Red high proper-motion objects in Tycho-2 and 2MASS

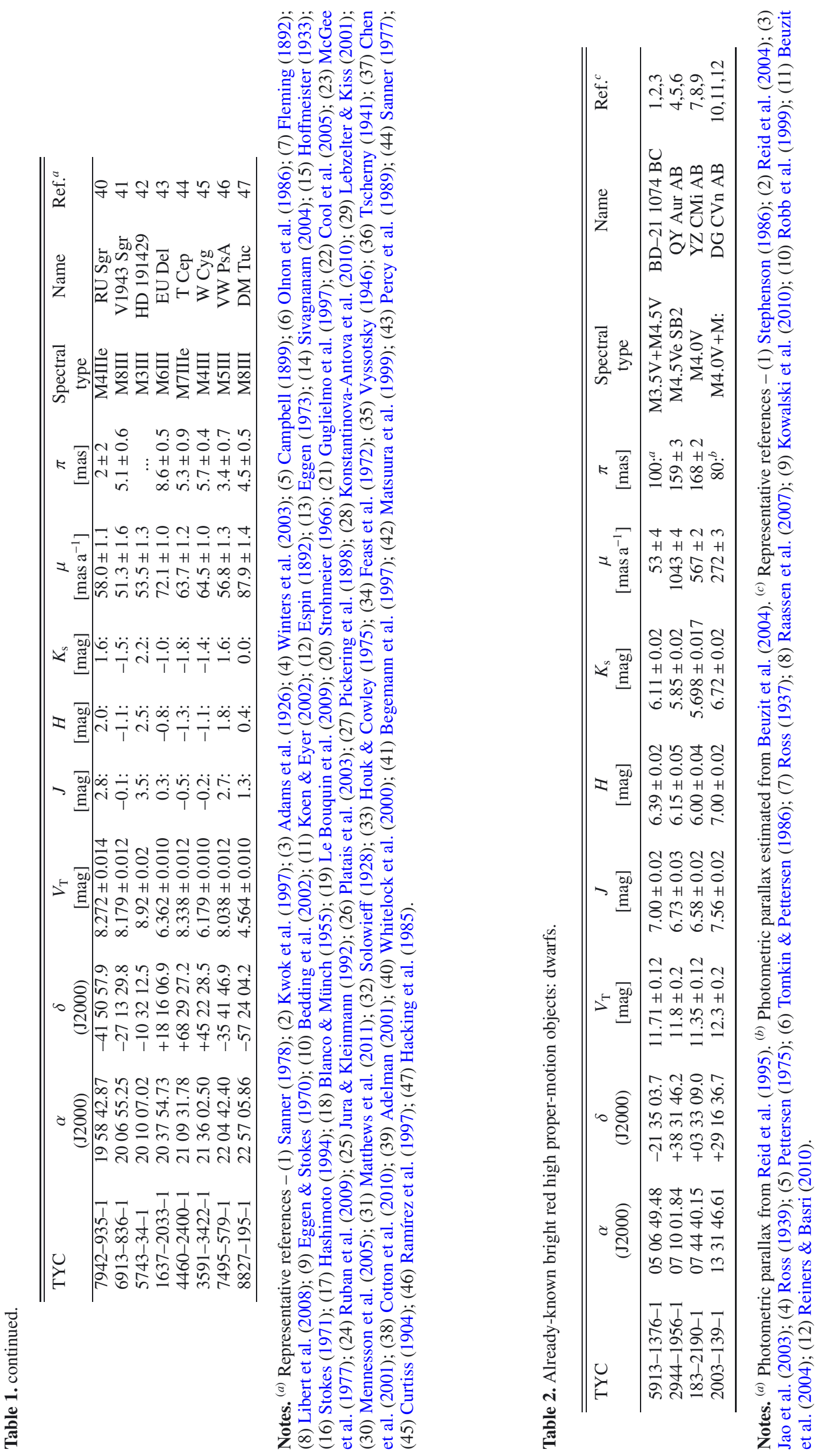


Table 3. New bright red high proper-motion objects ${ }^{a}$.

\begin{tabular}{|c|c|c|c|c|c|c|}
\hline Ruber & $\begin{array}{c}\bar{\lambda} \\
{[\mu \mathrm{m}]}\end{array}$ & 4 & $\begin{array}{c}5 \\
\text { (BD-19 4369) }\end{array}$ & 6 & 7 & 8 \\
\hline TYC & & 8299-2512-1 & $6211-498-1$ & $6211-472-1$ & $5648-545-1$ & 5113-1090-1 \\
\hline IRAS & & $15223-4834$ & R16234-1931 & $16249-1919$ & 17099-0950 & R18397-0123 \\
\hline$\alpha(\mathrm{J} 2000)$ & & $15: 25: 56.87$ & $16: 26: 21.17$ & $16: 27: 50.71$ & $17: 12: 40.72$ & $18: 42: 20.54$ \\
\hline$\delta(\mathrm{J} 2000)$ & & $-48: 44: 35.1$ & $-19: 38: 43.9$ & $-19: 26: 07.1$ & $-09: 54: 12.1$ & $-01: 20: 15.2$ \\
\hline$l(\mathrm{deg})$ & & 327.42 & 356.85 & 357.26 & 12.09 & 30.73 \\
\hline$b(\operatorname{deg})$ & & +6.64 & +20.03 & +19.90 & +16.73 & +1.47 \\
\hline$\mu_{\alpha} \cos \delta\left[\mathrm{mas} \mathrm{a}^{-1}\right]$ & & $+61 \pm 3$ & $-58 \pm 3$ & $-117 \pm 3$ & $-31 \pm 3$ & $-33 \pm 2$ \\
\hline$\mu_{\delta}\left[\mathrm{mas} \mathrm{a}^{-1}\right]$ & & $-8 \pm 3$ & $-2 \pm 3$ & $-63 \pm 4$ & $-40 \pm 3$ & $-47 \pm 2$ \\
\hline$H_{V_{\mathrm{T}}}[\mathrm{mag}]$ & & $10.04 \pm 0.18$ & $10.08 \pm 0.18$ & $11.89 \pm 0.13$ & $9.60 \pm 0.19$ & $9.64 \pm 0.15$ \\
\hline \multicolumn{7}{|c|}{ Photometric data } \\
\hline Tycho- $2 B_{\mathrm{T}}[\mathrm{mag}]$ & 0.42 & $12.8 \pm 0.3$ & $14.3 \pm 0.6$ & $13.9 \pm 0.5$ & $12.9 \pm 0.3$ & $12.4 \pm 0.2$ \\
\hline Tycho- $2 V_{\mathrm{T}}[\mathrm{mag}]$ & 0.53 & $11.07 \pm 0.09$ & $11.26 \pm 0.10$ & $11.27 \pm 0.10$ & $11.08 \pm 0.09$ & $10.82 \pm 0.08$ \\
\hline UCAC3 $r$ [mag] & 0.63 & $10.22 \pm 0.02$ & $10.52 \pm 0.06$ & 10.62: & $10.30 \pm 0.02$ & $10.035 \pm 0.013$ \\
\hline DENIS $i$ [mag] & 0.80 & $8.84 \pm 0.04$ & $9.25 \pm 0.04$ & $\ldots$ & $8.37 \pm 0.02$ & $8.80 \pm 0.05$ \\
\hline 2MASS $J$ [mag] & 1.23 & $6.52 \pm 0.02$ & $6.82 \pm 0.02$ & $6.61 \pm 0.02$ & $6.192 \pm 0.018$ & $6.184 \pm 0.019$ \\
\hline 2MASS $H[\mathrm{mag}]$ & 1.66 & $5.56 \pm 0.02$ & $5.94 \pm 0.04$ & $5.66 \pm 0.03$ & $5.22 \pm 0.04$ & $5.15 \pm 0.03$ \\
\hline $2 \mathrm{MASS} K_{\mathrm{s}}[\mathrm{mag}]$ & 2.16 & $5.191 \pm 0.017$ & $5.625 \pm 0.017$ & $5.31 \pm 0.03$ & $4.81 \pm 0.02$ & $4.81 \pm 0.02$ \\
\hline WISE $W 1$ [mag] & 3.35 & $5.05 \pm 0.06$ & $5.49 \pm 0.05$ & $5.16 \pm 0.06$ & $4.76 \pm 0.06$ & $4.69 \pm 0.06$ \\
\hline WISE W2 [mag] & 4.60 & $4.88 \pm 0.04$ & $5.24 \pm 0.03$ & $4.70 \pm 0.03$ & $4.32 \pm 0.03$ & $4.28 \pm 0.04$ \\
\hline GLIMPSE 5.8 [mag] & 5.76 & $\ldots$ & $\ldots$ & $\ldots$ & $\ldots$ & $4.59 \pm 0.03$ \\
\hline GLIMPSE 8.0 [mag] & 7.96 & $\ldots$ & $\ldots$ & $\ldots$ & $\ldots$ & $4.58 \pm 0.02$ \\
\hline$M S X A[\mathrm{Jy}]$ & 8.28 & & & $\ldots$ & $\cdots$ & $0.85 \pm 0.04$ \\
\hline AKARI $S 09$ [Jy] & 9.22 & $0.581 \pm 0.009$ & $0.437 \pm 0.014$ & . & $0.81 \pm 0.02$ & $0.82 \pm 0.04$ \\
\hline WISE $W 3$ [mag] & 11.56 & $4.92 \pm 0.02$ & $5.36 \pm 0.02$ & $5.08 \pm 0.02$ & $4.62 \pm 0.02$ & $4.63 \pm 0.02$ \\
\hline IRAS 12 [Jy] & 11.60 & $0.49 \pm 0.04$ & $\ldots$ & $0.38 \pm 0.05$ & $0.77 \pm 0.18$ & \\
\hline AKARI $S 18$ [Jy] & 19.81 & $0.15 \pm 0.03$ & $\ldots$ & $\ldots$ & $0.23 \pm 0.07$ & $0.208 \pm 0.003$ \\
\hline WISE W4 [mag] & 22.09 & $4.83 \pm 0.03$ & $5.31 \pm 0.03$ & $5.00 \pm 0.03$ & $4.53 \pm 0.03$ & $4.61 \pm 0.03$ \\
\hline \multicolumn{7}{|c|}{ Estimated parameters $^{b}$} \\
\hline$T_{\text {eff }}[\mathrm{K}]$ & & $3400 \pm 100$ & $2900 \pm 100$ & $3000 \pm 100$ & $3100 \pm 100$ & $3200 \pm 100$ \\
\hline $\log g[\mathrm{cgs}]$ & & $3.5 \pm 0.5$ & $3.5 \pm 0.5$ & $3.5 \pm 0.5$ & $3.5 \pm 0.5$ & $4.0 \pm 0.5$ \\
\hline$P[\mathrm{~d}]$ & & $42.3 \pm 0.3$ & $(4000)$ & $2200 \pm 800$ & $420 \pm 30$ & $(7000)$ \\
\hline Amplitude [mmag] & & $126 \pm 9$ & $(50)$ & $70 \pm 40$ & $80 \pm 50$ & (60) \\
\hline Sp. type & & $\ldots$ & $\ldots$ & $\ldots$ & M2III: & M2III: wk \\
\hline
\end{tabular}

Notes. ${ }^{(a)}$ Tycho-2 and IRAS identification, equatorial coordinates from 2MASS, Galactic coordinates and proper motions from Tycho-2; magnitudes and fluxes from catalogues listed in the text; $T_{\text {eff }}$ and $\log g$ from our SED fits; period and amplitude in the $V$ band from our time-series analysis; and spectral type from our CAFOS observations. ${ }^{(b)}$ Tentative periods and amplitudes in parenthesis.

and $3400 \mathrm{~K}$ and between 3.5 and 4.0, respectively (see the bottom of Table 3 ). The accuracy in determining these parameters was set by the model grid size to $100 \mathrm{~K}$ in $T_{\text {eff }}$ and 0.5 in $\log g$. Estimated temperatures and surface gravities correspond to latetype $M$ stars with $\log g$ midway between dwarfs and giants. In Fig. 3 we plot the theoretical fitting of the observational SEDs. A VOSA upgrade, with new grids of theoretical isochrones for dwarf and giant stars with lower $T_{\text {eff }}$ s than those provided by the Kurucz models and wide intervals of metallicity, is currently under development by the Spanish Virtual Observatory team but, unfortunately, was not available to us at the time of writing. Nevertheless, the effect of the metallicity on the overall SEDs of dwarfs would probably be not strong enough to produce a noticeable change in the $T_{\text {eff }}$ and $\log g$ fit values, especially when fitting broad-band photometry (Bonfils et al. 2005; Rojas-Ayala et al. 2011; Schlaufman \& Laughlin 2010; Woolf et al. 2009).

\subsubsection{Photometric variability}

Another piece of information that may help in classifying the five Ruber stars in Table 3 is their photometric variability, which is often detected in red giant stars and in magnetically-active field M dwarfs. In general, active $\mathrm{M}$ dwarfs rotate fast (Stauffer \& Hartmann 1986; Bouvier et al. 1993; Delfosse et al. 1998; Rockenfeller et al. 2006), while giants pulsate with long periods (Stebbins \& Huffer 1930; Percy \& Polano 1998; Koen \& Eyer 2002; Catelan 2009). Periods, if detected, may also help differentiate the two kinds of objects.

We searched in the All Sky Automated Survey (ASAS) catalogue of variable stars (Pojmański 2002) for data on our objects. One of them, Ruber 4 (ASAS 152557-4844.6), was catalogued to have a period of photometric variability $P=43 \mathrm{~d}$ with an amplitude of $0.30 \mathrm{mag}$ in the $V$ band. Tabulated variability type was "MISC/SR" ("mostly semi-regular" with time scales of variation between 10 and $200 \mathrm{~d}$ ).

To do our own time-series analysis, we looked for the original light-curve data set of the five Ruber targets at the ASAS webpage $^{5}$ as in Caballero et al. (2010). The light curves, which contain approximately between 400 and 700 data points after discarding low-quality measurements, span from February 2001 to October 2009 and are displayed in Fig. 4. By eye, most stars

5 http://www.astrouw.edu.pl/asas/ 


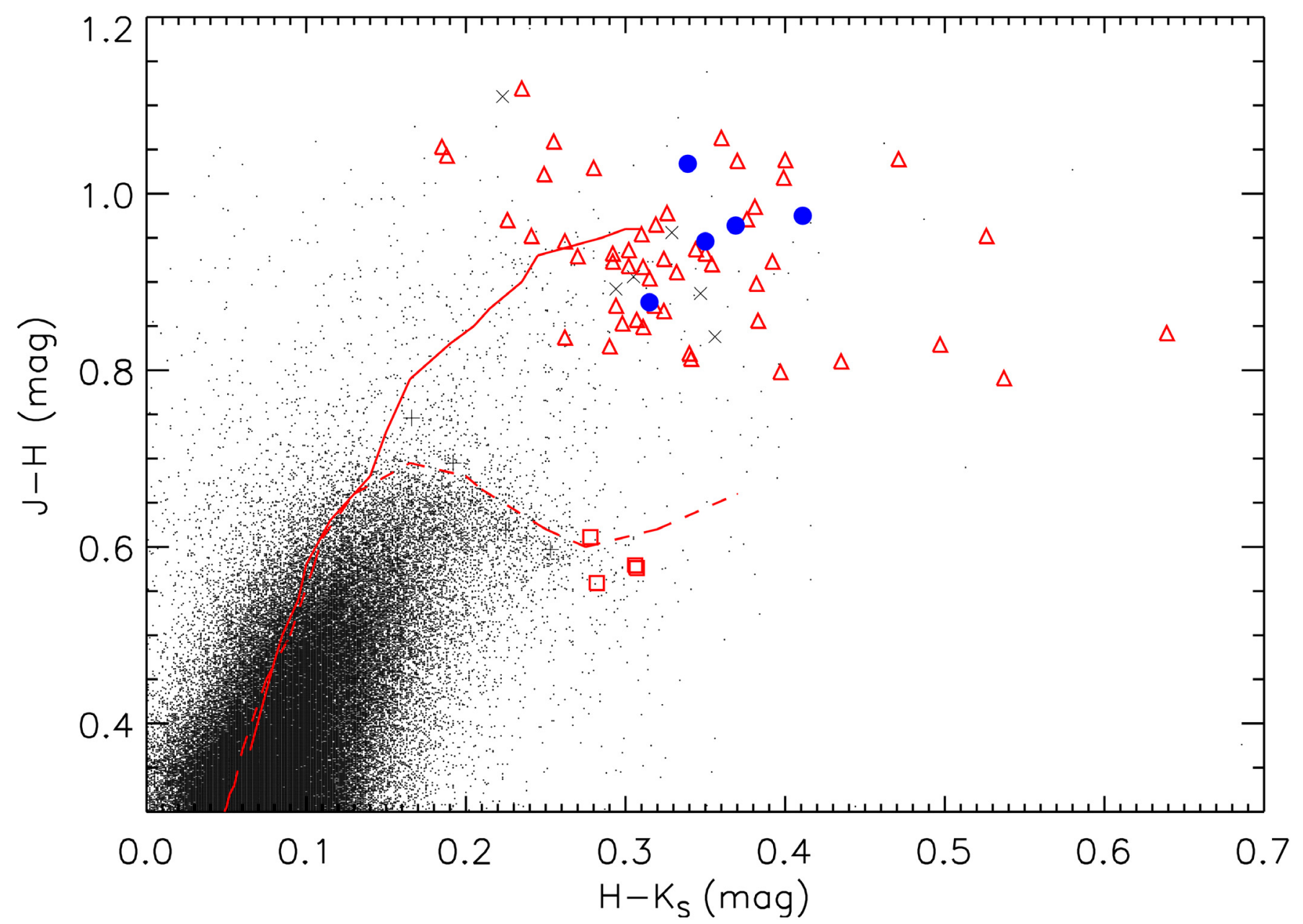

Fig. 2. Same as Fig. 1 but for the near-infrared colour-colour diagram. Dashed and continuous lines depict the intrinsic colours of M dwarfs and giants, respectively (Bessell \& Brett 1988). The two reddest dwarfs, QY Aur AB and YZ CMi AB, have almost coincident near-infrared colours.

display amplitudes of variability between two and three times the uncertainty of the photometry. In particular, for Ruber 4, which is the most variable star in our small sample, the mean error bar and standard deviation of the light curve are 40 and $116 \mathrm{mmag}$, respectively. The photometric variability (Tycho-2, 2MASS, ASAS data are not coincident in time) cannot explain the abnormal observed $V_{\mathrm{T}}-K_{\mathrm{s}}$ colours with respect to the reduced proper motions.

We performed the same variability analysis as in Jiménez-Esteban et al. (2006), using a sinusoidal light curve as a first approximation of the real one. The least mean square method (Stellingwerf 1978) was applied for a range of periods from $0.1 \mathrm{~d}$ to $10000 \mathrm{~d}$, and the quality of the fit was determined with a normalised $\chi^{2}$ test, weighting each observation with the inverse of the square of the observational error.

The periodograms for Ruber 4 to 8 are shown in Fig. 5. As illustrated by the first panel, apart from confirming the Pojmański (2002) period of Ruber 4 within uncertainties $(P=42.3 \pm 0.1 \mathrm{~d})$, we report on the discovery of a long secondary period (LSP) at around $520 \mathrm{~d}$. A similar period $(P \sim 420 \mathrm{~d})$ was found for Ruber 7, while Ruber 6 displayed a period longer than half the total time coverage of 3150-3200d (cf. bottom of Table 3). For Ruber 5 and Ruber 8, two extremely long periods were found ( $P \sim 4000$ and $\sim 7000 \mathrm{~d}$, respectively). Since they are much more longer than the time coverage of the monitoring, they should be considered highly tentative so need to be confirmed. All periodograms show a similar pattern around $300-400 \mathrm{~d}$, which is due to the frequency of the ASAS observations, with blocks of data separated by about a year.

The presence of LSPs, as in the case of Ruber 4, is very common among pulsating red giant stars. At least one third of the semi-regular variables in the solar vicinity are in fact multiperiodic, with an LSP roughly one order of magnitude longer than the primary period of pulsation (Wood et al. 2004; Percy et al. 2004). In spite of that, LSP is the only type of large-amplitude stellar variability that remains completely unexplained. Wood et al. (2004) discuss several mechanisms, but find no clear explanation for any, and speculate that asymptotic giant branch stars with LSP may be the precursors of asymmetric planetary nebulae. More recent studies (Soszyński et al. 2007; Soszyński 2007) have pointed towards a binary origin. This last explanation is consistent with the location of Ruber 4 in the reduced proper motion diagram, where other multiple stellar system are found.

Another peculiar object is Ruber 6, which presents an extremely long period $(P \sim 2200 \mathrm{~d})$ with low-amplitude variability $(\sim 0.07 \mathrm{mag})$, and relatively blue NIR colour $\left(H-K_{\mathrm{s}} \sim 0.35 \mathrm{mag}\right)$. We did not find any other star with a longer period reported in the bibliography. Two kinds of red giant stars could present such long periods: Mira-like variables (Engels et al. 1983; JiménezEsteban et al. 2006) and semi-regular variables (e.g. Glass \& van Leeuwen 2007, and references therein). In the first case, stars present very red NIR colours $\left(H-K_{\mathrm{S}}>3 \mathrm{mag}\right)$ and a large 


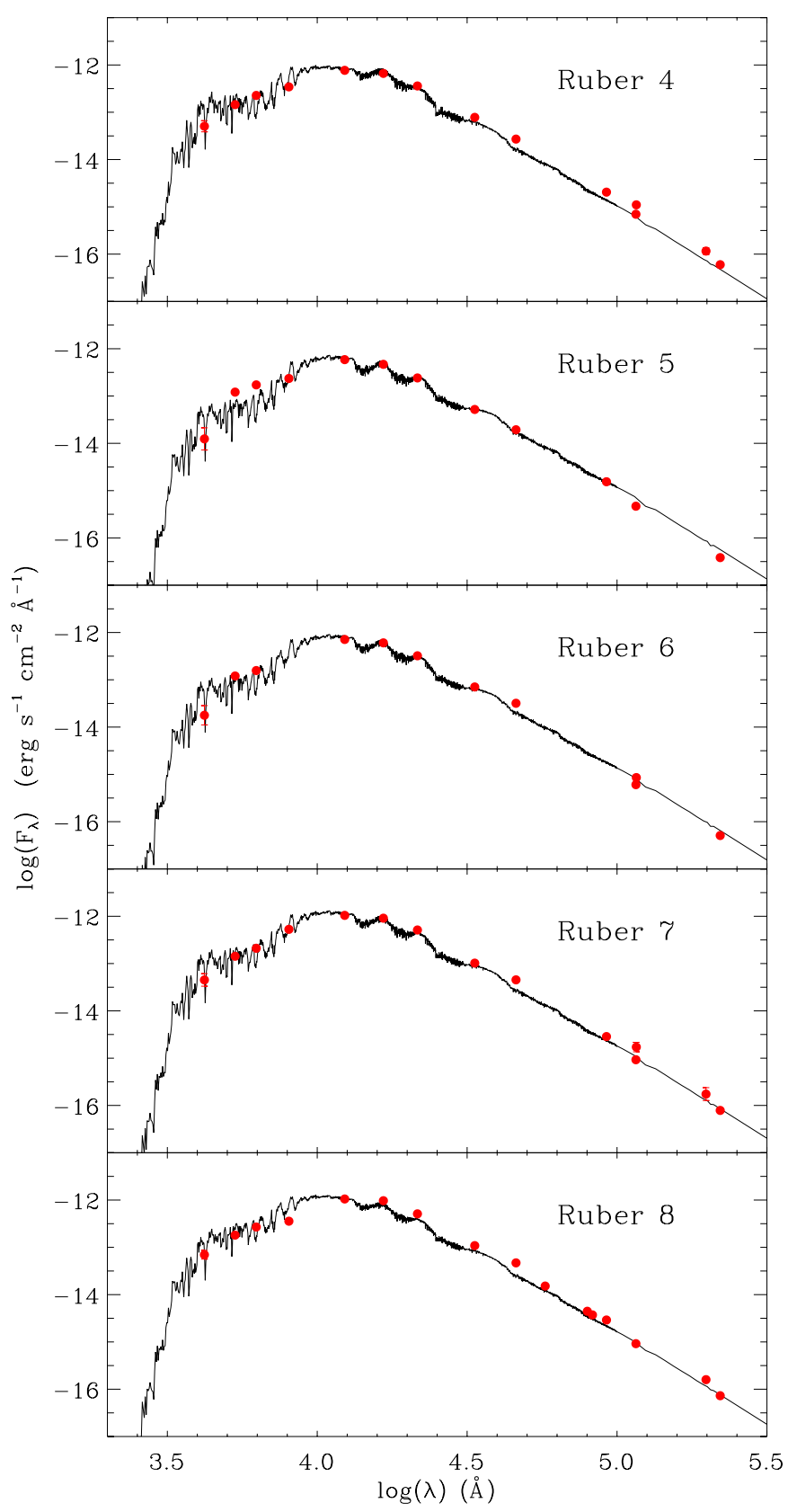

Fig. 3. Theoretical SED fits of Ruber 4 to 8, from top to bottom. Solid lines represent the NextGen models for solar metallicity that best fit the observational data. Filled [red] circles indicate the observational photometric data used for the fit. Error bars are usually smaller than the size of the circles.

amplitude of variability ( $>1 \mathrm{mag}$ ); in the second case, the stars have bluer colours and present lower amplitudes. Ruber 6 may be a semi-regular variable star with an extremely long period and very low-amplitude variability.

\subsection{Spectroscopy}

The best way to ascertain the nature of the Ruber 4 to 8 sources is with spectroscopic data. Consequently, we used another VO tool, VOSED ${ }^{6}$ (Gutiérrez et al. 2008), to look for spectra in the VO archives. Developed by the Spanish Virtual Observatory,

${ }^{6}$ http://sdc.cab.inta-csic.es/vosed

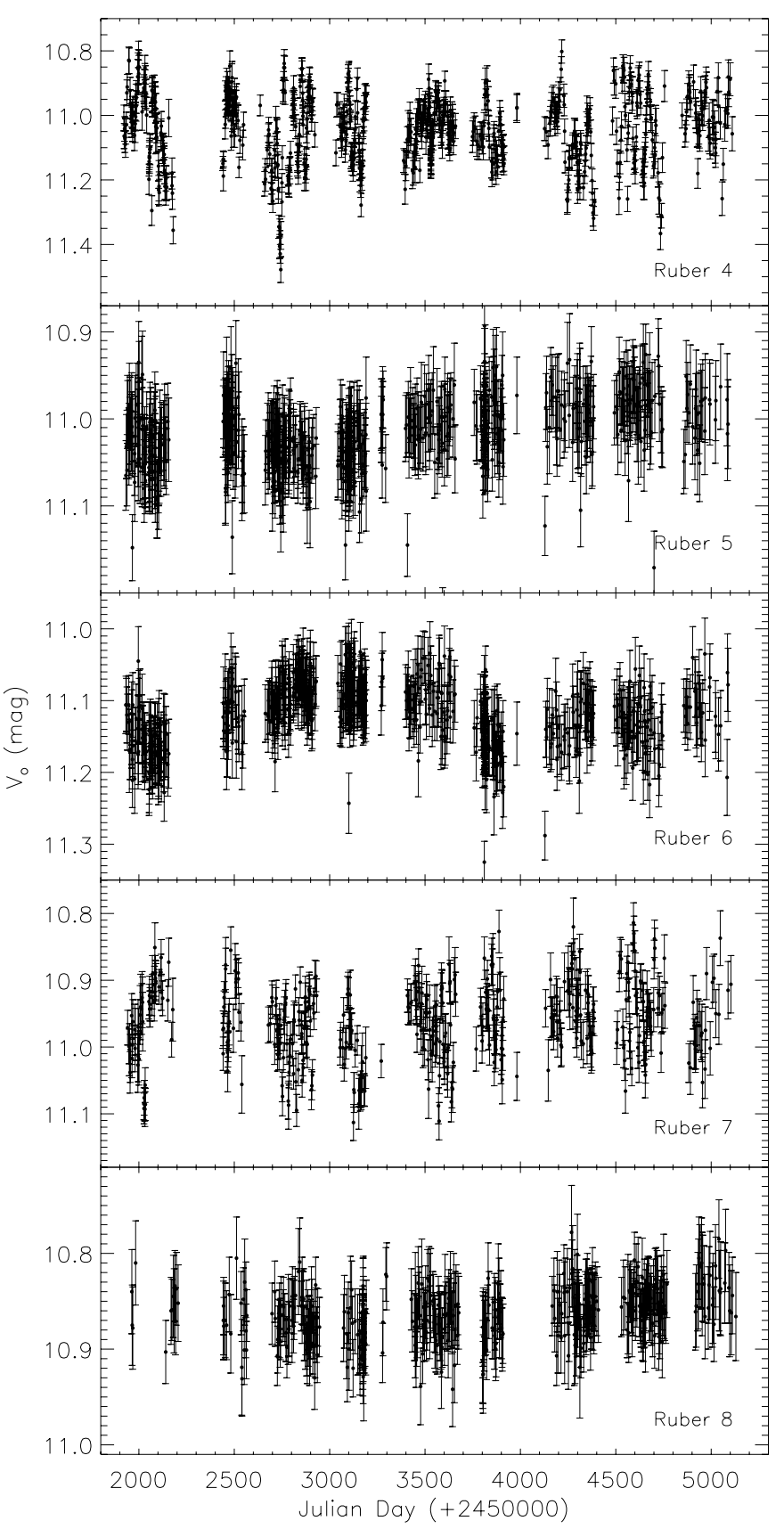

Fig. 4. ASAS $V$-band light curves of Ruber 4 to 8 , from top to bottom.

VOSED allows the user to gather spectroscopic information available throughout the VO. Unfortunately, no spectroscopic data were found.

To complement our VO analysis, we collected lowresolution optical spectra for Ruber 7 and 8, TYC 6238-480-1 (the discarded low-proper motion giant in Sect. 2.1) and eleven comparison stars with a ground telescope. On 2011 Mar 20, we used the Calar Alto Faint Object Spectrograph ${ }^{7}$ (CAFOS) at the $2.2 \mathrm{~m}$ telescope on the Calar Alto Observatory, Almería, Spain, with the grism Green-100 and the SITe $1 \mathrm{~d}$ detector of $24 \mu \mathrm{m}$ pixels. The resulting resolution was only $R \sim 1600$, but the wavelength coverage was very wide, from 4900 to $7800 \AA$ without vignetting. Needed exposure times were in the range of 100-200 s.

7 http://www.caha.es/alises/cafos/cafos.html 


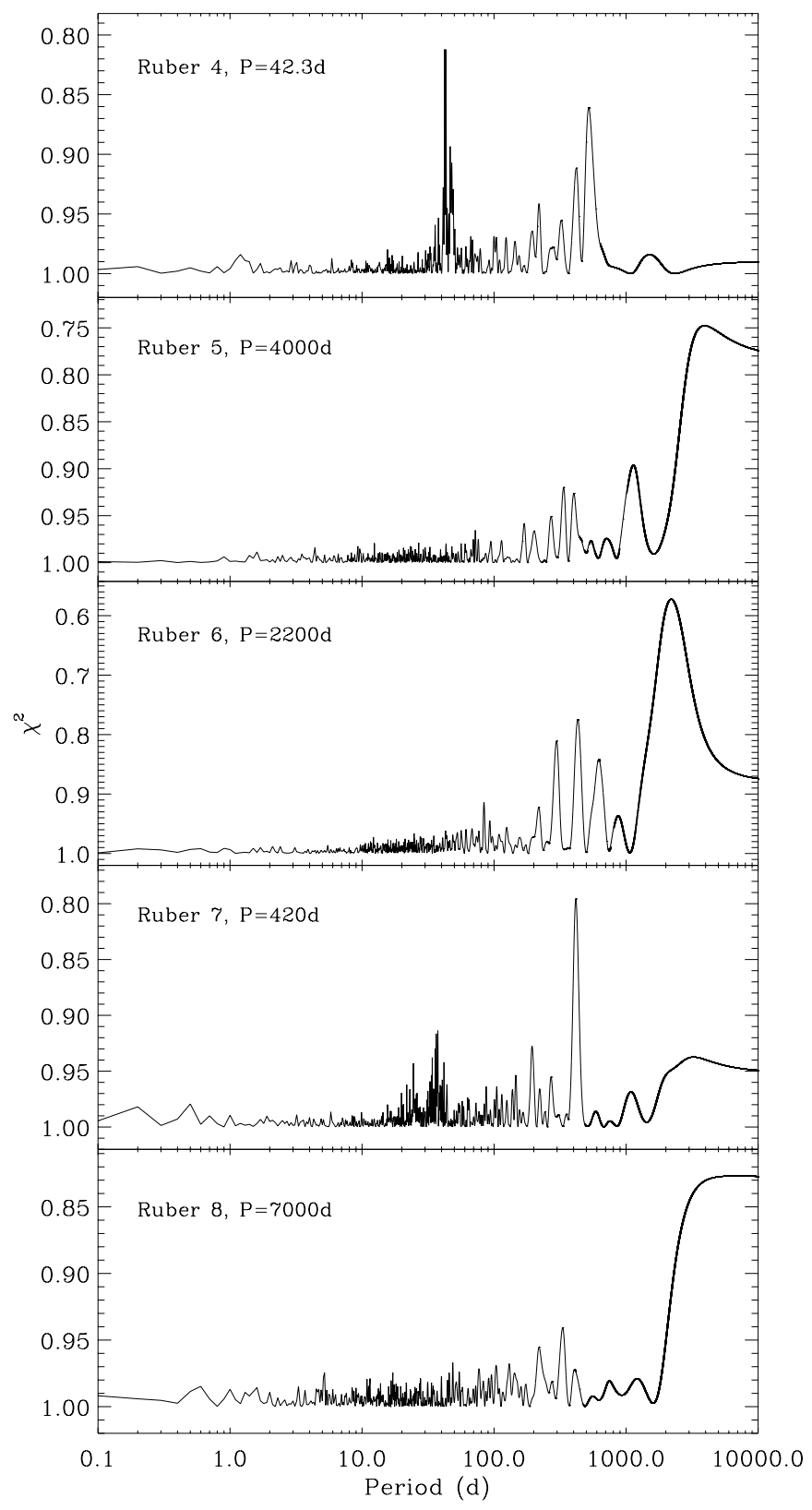

Fig. 5. Periodograms of the ASAS light curved of Ruber 4 to 8 , from top to bottom.

No other Ruber stars could be observed because of their low declination for Calar Alto.

The eleven comparison stars were seven nearby late-type dwarfs and four M-type giants. We reduced, extracted, and corrected all the spectra for instrumental response (with the standard star Feige 34) using standard tasks within the IRAF environment. The fourteen spectra are shown in Fig. 7.

From the visual inspection of the pseudocontinuum of the CAFOS spectra, we concluded that both Ruber 7 and 8 are in the giant class. Therefore, we estimated their spectral types from the comparison with the four other observed giants. Unfortunately, their spectral type determinations do not seem to be fully reliable: the spectra of $\mathrm{BW} C V n$ and $\mathrm{BZ} \mathrm{CVn}$ are identical, but the reported spectral types are different (M1III vs. M3III). Something similar happens to HD 98500 and HD 122132. After consulting additional M giant standards in Danks \& Dennefeld (1994), we gave the

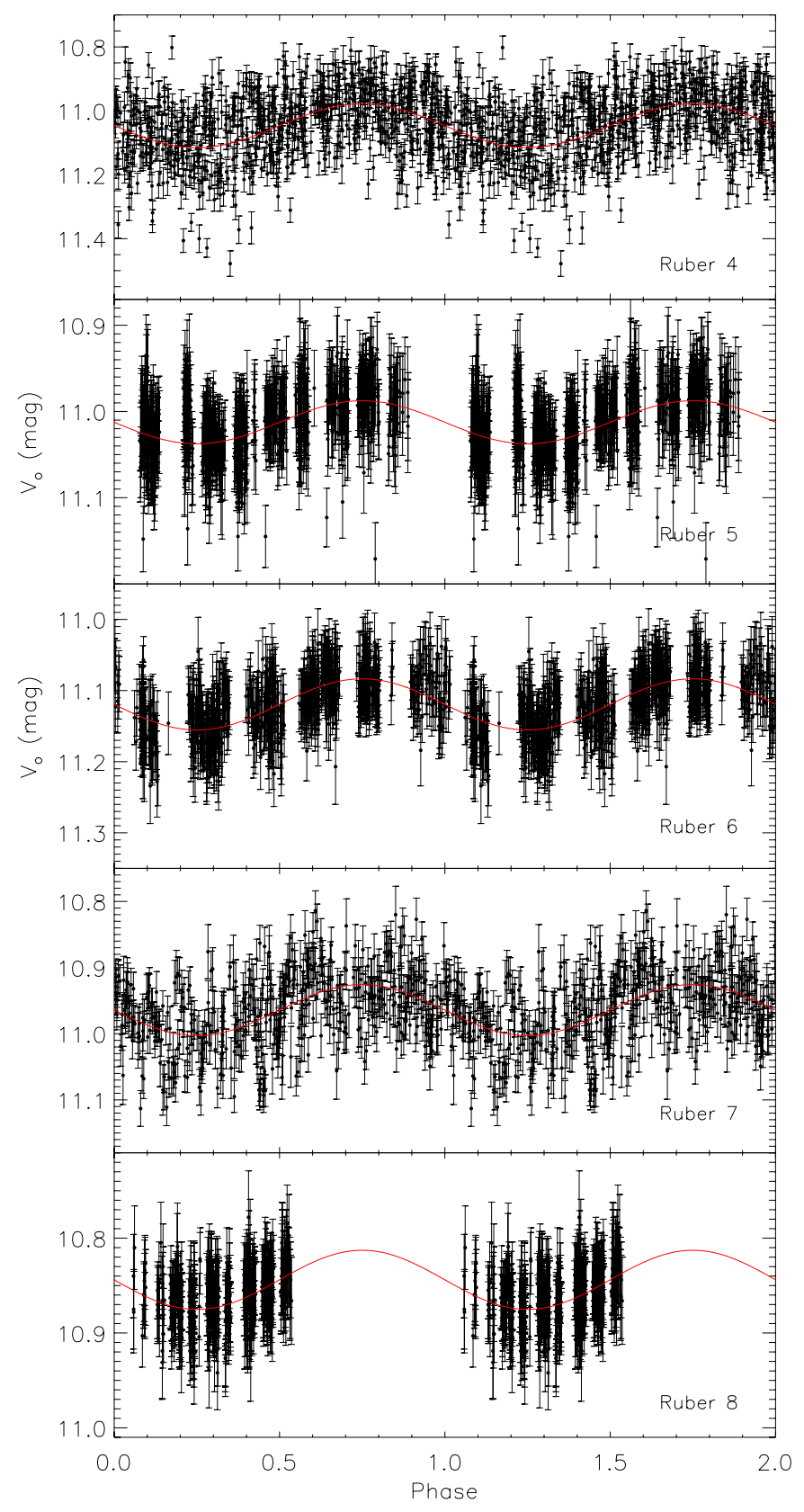

Fig. 6. ASAS $V$-band light curves as a function of phase of Ruber 4 to 8, from top to bottom. Continue (red) lines show the corresponding symmetric sinusoidal model light curve fit. Light curves of Ruber 5 and 8 are highly tentative.

M2III spectral type, with one dex uncertainty, to both Ruber 7 and TYC 6238-480-1. However, the spectrum of Ruber 8 deserved further attention.

The pseudocontinuum of Ruber 8 reasonably fits that of other M2 giants. However, its absorption bands and (alkali) lines are far less marked, so we gave it the "wk" code of spectral peculiarity (from "weak lines"). This weakness of bands and lines may be explained by a very low metallicity. Metal-poor stars are thought to be the survivors of the earliest generations of stars. Their study helps to put constraints, for example, on the chemical history of the Milky Way (Hollek et al. 2011; Cayrel 2006). Bright low-metallicity stars are very rare, so if the metal-poor nature of Ruber 8 is confirmed, it would become an excellent target for detailed follow-up studies. Until now, we have failed to obtain a higher resolution spectrum of Ruber 8, from which we 

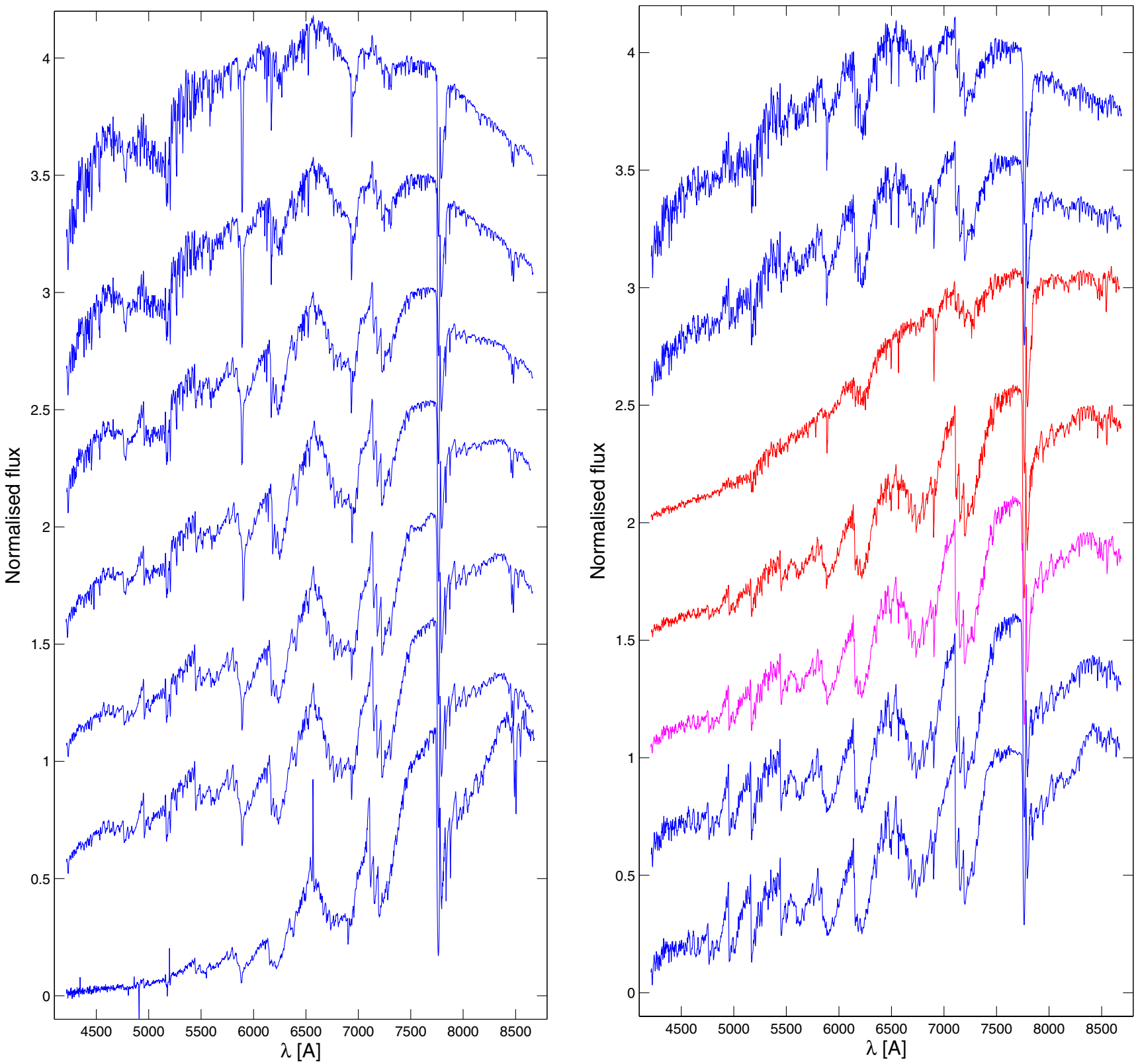

Fig. 7. CAFOS spectra of Ruber 7 and 8, TYC 6238-480-1, and eleven comparison stars. Left panel (dwarfs): from top to bottom, BD+39 2801 (K5V), HD 147379 A (K7V), GJ 1170 (M1V), HD 95735 (M2V), GJ 4040 (M3V), GJ 687 (M3V), and GJ 1101 (M3.5Ve). All the spectral types are from Hawley et al. (1996). Right panel (giants): from top to bottom, HD 98500 (M0III; Moore \& Paddock 1950), HD 122132 (M2III; Moore \& Paddock 1950), Ruber 8 [in red], Ruber 7 [in red], TYC 6238-480-1 [in magenta], BW CVn (M1III; Upgren 1960), and BZ CVn (M3III; Schild 1973).

would measure radial velocities, determine galactocentric space velocities $U V W$, and assign membership for the Galactic kinematic components (i.e. thin/thick disc, halo).

\section{Summary and final remarks}

We identified red high proper-motion objects in the Tycho-2 and 2MASS catalogues using Virtual Observatory tools, in the same way as Jiménez-Esteban et al. (2011) did with blue objects. After discarding six sources with erroneous proper motions and eight close binaries, we got a sample of 59 objects with proper motions

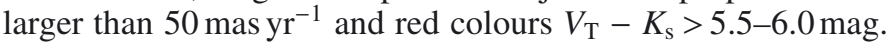
Of them, 54 were known to be M-type giants and dwarfs. The other five, namely Ruber 4 to 8 , are studied in this work for the first time.
We collected and analysed all available data of the Ruber objects. From SED theoretical fits, we estimated temperatures that correspond to late-type M stars and surface gravities midway between dwarfs and giants (subject to uncertainties in the used models), but from NIR colours, we concluded that all of them are likely red giants with high tangential velocities.

The analysis of their ASAS light curves yielded interesting results. We established reliable periods for three of the five Ruber objects. In the case of Ruber 4, we also found a secondary period that is roughly ten times longer than the primary one. Ruber 6 presents an extremely long period $(P \sim 2200 \mathrm{~d})$, although with a large error due to the short time coverage. Ruber 5 and 8 present even longer periods, but they are uncertain.

With the help of low-resolution spectra obtained with CAFOS on Calar Alto, we determined the spectral type of 
Ruber 7 and 8 at M2III: and M2III: wk, respectively. The spectrum of Ruber 8 displays low marked absorption bands and lines, probably due to a metal-poor nature.

Because of their brightness, the five Ruber objects can serve as useful targets for detailed studies of old-population giants.

Acknowledgements. We are in debt to D. Montes for supervising the spectroscopic observations on Calar Alto, to A. Klutsch for trying to get additional spectra for Ruber objects, and to C. Rodrigo for clarifying which models are actually available in VOSA. This research made use of VOSA and VOSED, developed by the Spanish Virtual Observatory through grants AyA2008-02156 and RI031675, and of Aladin and SIMBAD developed at the Centre de Données astronomiques de Strasbourg, France. Financial support was provided by the Spanish Ministerio de Ciencia e Innovación, Universidad Complutense de Madrid and Comunidad Autónoma de Madrid under grants CSD2006-00070 (under the Consolider-Ingenio 2010 Programme First Science with the Gran Telescopio Canarias), AyA2008-00695, AyA2008-06423-C0303, and SP2009/ESP-1496. This research made use of the NASA/IPAC Infrared Science Archive, which is operated by the Jet Propulsion Laboratory, California Institute of Technology, under contract with the National Aeronautics and Space Administration. Based on observations collected at the Centro Astronómico Hispano Alemán (CAHA) at Calar Alto, operated jointly by the Max-PlanckInstitut für Astronomie and the Instituto de Astrofísica de Andalucía (CSIC).

\section{References}

Adams, W. S., Joy, A. H., \& Humason, M. L. 1926, ApJ, 64, 225 Adelman, S. J. 2001, Baltic Astron., 10, 589

Bayo, A., Rodrigo, C., Barrado y Navascués, D., et al. 2008, A\&A, 492, 277

Bedding, T. R., Zijlstra, A. A., Jones, A., et al. 2002, MNRAS, 337, 79

Beers, T. C., Drilling, J. S., Rossi, S., et al. 2002, AJ, 124, 931

Begemann, B., Dorschner, J., Henning, T., et al. 1997, ApJ, 476, 199

Beichman, C. A., Neugebauer, G., Habing, H. J., Clegg, P. E., \& Chester,

T. J. 1988, in Infrared astronomical satellite (IRAS) catalogs and atlases, Explanatory Suppl., 1

Benz, A. O., \& Alef, W. 1991, A\&A, 252, L19

Bergeat, J., Knapik, A., \& Rutily, B. 2002, A\&A, 390, 987

Bessell, M. S., \& Brett, J. M. 1988, PASP, 100, 1134

Beuzit, J.-L., Ségransan, D., Forveille, T., et al. 2004, A\&A, 425, 997

Blanco, V. M., \& Münch, L. 1955, Bol. Observ. Tonantzintla y Tacubaya, 2, 17

Bonfils, X., Delfosse, X., Udry, S., et al. 2005, A\&A, 442, 635

Bonnarel, F., Fernique, P., Bienaymé, O., et al. 2000, A\&AS, 143, 33

Bouvier, J., Cabrit, S., Fernández, M., Martín, E. L., \& Matthews, J. M. 1993, A\&A, 272, 176

Caballero, J. A. 2009, Multi-wavelength Astronomy and Virtual Observatory, Proceedings of the EURO-VO Workshop, held at the European Space Astronomy Centre of ESA, Villafranca del Castillo, Spain, 1-3 December, 2008, ed. D. Baines, \& P. Osuna (The European Space Agency), 3

Caballero, J. A. 2010, A\&A, 514, A18

Caballero, J. A., \& Solano, E. 2008, A\&A, 485, 931

Caballero, J. A., Cornide, M., \& de Castro, E. 2010, Astron. Nachr., 331, 257

Campbell, W. W. 1899, ApJ, 9, 31

Carney, B. W., \& Latham, D. W. 1986, AJ, 92, 60

Catelan, M. 2009, Ap\&SS, 320, 261

Cayrel, R. 2006, Rep. Prog. Phys., 69, 2823

Chen, P. S., Szczerba, R., Kwok, S., \& Volk, K. 2001, A\&A, 368, 1006

Churchwell, E., Babler, B. L., Meade, M. R., et al. 2009, PASP, 121, 213

Cool, R. J., Howell, S. B., Peña, M., Adamson, A. J., \& Thompson, R. R. 2005, PASP, 117,462

Cotton, W. D., Ragland, S., Pluzhnik, E. A., et al. 2010, ApJS, 188, 506

Curtiss, R. H. 1904, ApJ, 20, 232

da Silva, L., Torres, C. A. O., de La Reza, R., et al. 2009, A\&A, 508, 833

Danks, A. C., \& Dennefeld, M. 1994, PASP, 106, 382

Delfosse, X., Forveille, T., Perrier, C., \& Mayor, M. 1998, A\&A, 331, 581

Delorme, P., Delfosse, X., Albert, L., et al. 2008, A\&A, 482, 961

Donner. 1935, MmRAS, 65, 51

Egan, M. P., Price, S. D., \& Kraemer, K. E. 2003, in The Midcourse Space

Experiment (MSX) Point Source Catalog Version 2.3

Eggen, O. J. 1973, MNRAS, 164, 423

Eggen, O. J., \& Stokes, N. R. 1970, ApJ, 161, 199

Engels, D., Kreysa, E., Schultz, G. V., \& Sherwood, W. A. 1983, A\&A, 124, 123

Epchtein, N., de Batz, B., Capoani, L., et al. 1997, The Messenger, 87, 27

Espin, T. E. 1892, Astron. Nachr., 129, 297

Famaey, B., Jorissen, A., Luri, X., et al. 2005, A\&A, 430, 165

Feast, M. W., Woolley, R., \& Yilmaz, N. 1972, MNRAS, 158, 23
Fleming, M. 1892, Astronomy and Astro-Physics (formerly The Sidereal Messenger), 11, 765

Glass, I. S., \& van Leeuwen, F. 2007, MNRAS, 378, 1543

Guglielmo, F., Epchtein, N., Arditti, F., \& Sevre, F. 1997, A\&AS, 122, 489

Gutiérrez, R., Solano, E., Delgado, A., Sarro, L. M., \& Merín, B. 2008, in Astronomical Spectroscopy and Virtual Observatory, ed. M. Guainazzi, \& P. Osuna, 91

Hacking, P., Neugebauer, G., Emerson, J., et al. 1985, PASP, 97, 616

Hashimoto, O. 1994, A\&AS, 107, 445

Hauschildt, P. H., Allard, F., \& Baron, E. 1999, ApJ, 512, 377

Hawley, S. L., Gizis, J. E., \& Reid, I. N. 1996, AJ, 112, 2799

Hoffmeister, C. 1933, Astron. Nachr., 247, 281

Høg, E., Fabricius, C., Makarov, V. V., et al. 2000, A\&A, 355, L27

Hollek, J. K., Frebel, A., Roederer, I. U., et al. 2011, ApJ, 742, 54

Houk, N., \& Cowley, A. P. 1975, University of Michigan Catalogue of twodimensional spectral types for the HD stars, Vol. I, Declinations $-90^{\circ}$ to $-53^{\circ}$ (Ann Arbor, USA: University of Michigan)

Ishihara, D., Onaka, T., Kataza, H., et al. 2010, A\&A, 514, A1

Jao, W.-C., Henry, T. J., Subasavage, J. P., et al. 2003, AJ, 125, 332

Jiménez-Esteban, F. M., García-Lario, P., Engels, D., \& Manchado, A. 2006, A\&A, 458, 533

Jiménez-Esteban, F. M., Caballero, J. A., \& Solano, E. 2011, A\&A, 525, A29

Jura, M., \& Kleinmann, S. G. 1992, ApJS, 83, 329

Kahler, S., Golub, L., Harnden, F. R., et al. 1982, ApJ, 252, 239

Kirkpatrick, J. D., Cushing, M. C., Gelino, C. R., et al. 2011, ApJS, 197, 19

Kiss, L. L., \& Bedding, T. R. 2003, MNRAS, 343, L79

Koen, C., \& Eyer, L. 2002, MNRAS, 331, 45

Konstantinova-Antova, R., Aurière, M., Charbonnel, C., et al. 2010, A\&A, 524, A57

Kowalski, A. F., Hawley, S. L., Holtzman, J. A., Wisniewski, J. P., \& Hilton, E. J. 2010, ApJ, 714, L98

Kundu, M. R., White, S. M., Jackson, P. D., \& Pallavicini, R. 1988, A\&A, 195, 159

Kunkel, W. E. 1969, Nature, 222, 1129

Kwok, S., Volk, K., \& Bidelman, W. P. 1997, ApJS, 112, 557

Lafrenière, D., Doyon, R., Marois, C., et al. 2007, ApJ, 670, 1367

Lambert, D. L., Gustafsson, B., Eriksson, K., \& Hinkle, K. H. 1986, ApJS, 62, 373

Le Bouquin, J.-B., Abuter, R., Haguenauer, P., et al. 2009, A\&A, 493, 747

Lebzelter, T., \& Kiss, L. L. 2001, A\&A, 380, 388

Lépine, S., \& Gaidos, E. 2011, AJ, 142, 138

Libert, Y., Le Bertre, T., Gérard, E., \& Winters, J. M. 2008, A\&A, 491, 789

Mahadevan, S., Ramsey, L., Wright, J., et al. 2010, in SPIE Conf. Ser., 7735

Matsuura, M., Yamamura, I., Murakami, H., Freund, M. M., \& Tanaka, M. 1999, A\&A, 348, 579

Matthews, L. D., Libert, Y., Gérard, E., et al. 2011, AJ, 141, 60

McGee, R. X., Newton, L. M., \& Brooks, J. W. 1977, MNRAS, 180, 91P

McWilliam, A., \& Rich, R. M. 1994, ApJS, 91, 749

Mennesson, B., Koresko, C., Creech-Eakman, M. J., et al. 2005, ApJ, 634, L169

Montes, D., López-Santiago, J., Gálvez, M. C., et al. 2001, MNRAS, 328, 45

Moore, J. H., \& Paddock, G. F. 1950, ApJ, 112, 48

Olnon, F. M., Raimond, E., Neugebauer, G., et al. 1986, A\&AS, 65, 607

Oreiro, R., Rodríguez-López, C., Solano, E., et al. 2011, A\&A, 530, A2

Percy, J. R., \& Polano, S. 1998, in A Half Century of Stellar Pulsation Interpretation, ed. P. A., Bradley, \& J. A., Guzik, ASP Conf. Ser., 135, 249

Percy, J. R., Landis, H. J., \& Milton, R. E. 1989, PASP, 101, 893

Percy, J. R., Bakos, A. G., Besla, G., et al. 2004, in IAU Colloq., 193, Variable Stars in the Local Group, ed. D. W., Kurtz, \& K. R., Pollard, ASP Conf. Ser., 310,348

Pettersen, B. R. 1975, A\&A, 41, 87

Pickering, E. C., Bailey, S. I., Fleming, W. P., Leland, E. F., \& Wells, L. D. 1898, ApJ, 7, 208

Platais, I., Pourbaix, D., Jorissen, A., et al. 2003, A\&A, 397, 997

Pojmański, G. 2002, Acta Astron., 52, 397

Quirrenbach, A., Amado, P. J., Mandel, H., et al. 2010, in SPIE Conf. Ser., 7735,

Raassen, A. J. J., Mitra-Kraev, U., \& Güdel, M. 2007, MNRAS, 379, 1075

Ramírez, S. V., Depoy, D. L., Frogel, J. A., Sellgren, K., \& Blum, R. D. 1997, AJ, 113, 1411

Reid, I. N., Hawley, S. L., \& Gizis, J. E. 1995, AJ, 110, 1838

Reid, I. N., Cruz, K. L., Allen, P., et al. 2004, AJ, 128, 463

Reiners, A., \& Basri, G. 2010, ApJ, 710, 924

Robb, R. M., Balam, D. D., \& Greimel, R. 1999, Information Bulletin on Variable Stars, 4714, 1

Rocha-Pinto, H. J., Flynn, C., Scalo, J., et al. 2004, A\&A, 423, 517

Rockenfeller, B., Bailer-Jones, C. A. L., \& Mundt, R. 2006, A\&A, 448, 1111

Rojas-Ayala, B., Covey, K. R., Muirhead, P. S., \& Lloyd, J. P. 2011, ApJ, submitted [arXiv: 1112.4567]

Ross, F. E. 1937, AJ, 46, 157 
A\&A 539, A86 (2012)

Ross, F. E. 1939, AJ, 48, 10

Ruban, E. V., Arkharov, A. A., Hagen-Thorn, E. I., \& Novikov, V. V. 2009, Astrophysics, 52, 383

Sanner, F. 1977, PASP, 89, 768

Sanner, F. 1978, ApJ, 219, 538

Schild, R. E. 1973, AJ, 78, 37

Schlaufman, K. C., \& Laughlin, G. 2010, A\&A, 519, A105

Schönfeld, E. 1886, in Bonner Durchmusterung des nordlichen Himmels, ed.

Marcus, Weber's Verlag, Bonn

Sivagnanam, P. 2004, MNRAS, 347, 1084

Skrutskie, M. F., Cutri, R. M., Stiening, R., et al. 2006, AJ, 131, 1163

Solowieff, A. W. 1928, Astron. Nachr., 233, 367

Soszyński, I. 2007, ApJ, 660, 1486

Soszyński, I., Dziembowski, W. A., Udalski, A., et al. 2007, Acta Astron., 57, 201

Stauffer, J. R., \& Hartmann, L. W. 1986, ApJS, 61, 531

Stebbins, J., \& Huffer, C. M. 1930, Publications of the Washburn Observatory, 15,140
Stellingwerf, R. F. 1978, ApJ, 224, 953

Stephenson, C. B. 1986, AJ, 91, 144

Stokes, N. R. 1971, MNRAS, 152, 165

Strohmeier, W. 1966, IBVS, 158,

Tomkin, J., \& Pettersen, B. R. 1986, AJ, 92, 1424

Tscherny, S. D. 1941, AJ, 49, 107

Upgren, A. R. 1960, AJ, 65, 644

van Leeuwen, F. 2007, A\&A, 474, 653

Vennes, S., Kawka, A., \& Németh, P. 2011, MNRAS, 410, 2095

Vyssotsky, A. N. 1946, PASP, 58, 54

Whitelock, P., Marang, F., \& Feast, M. 2000, MNRAS, 319, 728

Winters, J. M., Le Bertre, T., Jeong, K. S., Nyman, L.-Å., \& Epchtein, N. 2003, A\&A, 409, 715

Wood, P. R., \& Cahn, J. H. 1977, ApJ, 211, 499

Wood, P. R., Olivier, E. A., \& Kawaler, S. D. 2004, ApJ, 604, 800

Woolf, V. M., Lépine, S., \& Wallerstein, G. 2009, PASP, 121, 117

Wright, E. L., Eisenhardt, P. R. M., Mainzer, A. K., et al. 2010, AJ, 140, 1868

Zacharias, N., Finch, C., Girard, T., et al. 2010, AJ, 139, 2184 